Department of Mathematics

\title{
Well-posed and Stable Transmission Problems
}

\author{
Jan Nordström and Viktor Linders \\ LiTH-MAT-R--2017/15--SE
}


Department of Mathematics

Linköping University

S-581 83 Linköping 


\title{
Well-posed and Stable Transmission Problems
}

\author{
Jan Nordström ${ }^{\mathrm{a}}$, Viktor Linders ${ }^{\mathrm{a}}$ \\ ${ }^{a}$ Department of Mathematics, Computational Mathematics, Linköping University, \\ SE-581 83 Linköping, Sweden (jan.nordstrom@liu.se, viktor.linders@liu.se).
}

\begin{abstract}
We introduce the notion of a transmission problem to describe a general class of problems where different dynamics are coupled in time. Well-posedness and stability is analysed for continuous and discrete problems using both strong and weak formulations, and a general transmission condition is obtained. The theory is applied to several examples including the coupling of fluid flow models, multi-grid implementations, multi-block formulations and numerical filtering.
\end{abstract}

Keywords: Initial-boundary value problems; Transmission problems; Energy estimates; Well-posedness; Multi-block; Numerical Filter; Interpolation; Multi-grid; Summation-by-Parts; Stability

\section{Introduction and motivation}

In this paper we will introduce a general class of initial-boundary value problems coupled in time, which we refer to as transmission problems. This class includes any setting described by the following schematic:

1. The solution is governed by the dynamics $\mathcal{D}_{1}$ from time $t_{1}$ to time $t_{2}$,

2. At $t_{2}$, the solution is subject to an operation $\mathcal{X}$,

3. At later times, the solution is governed by the (possibly different) dynamics $\mathcal{D}_{2}$.

Central to this class is the transmission operator $\mathcal{X}$, which we assume admits a matrix representation, but is otherwise left completely general.

The aim of this paper is to obtain conditions for $\mathcal{X}$ under which the solution to the transmission problem is bounded by available data, in particular initial data available at time $t_{1}$; a prerequisite for well-posedness. However, boundedness of a solution depends on the norm in which it is estimated. This 
necessitates certain assumptions on the operators $\mathcal{D}_{1,2}$, and we will therefore confine the analysis to operators that are semi-bounded in a generalised $L^{2}$ norm $[1,2]$. In essence, this means that the transmission problem is amenable to analysis via the energy method.

We will consider continuous transmission problems where initial, boundary and coupling conditions are imposed either strongly, or weakly through so called lifting operators [3, 4]. It will be shown that energy boundedness is equivalent to a certain condition relating the operator $\mathcal{X}$ and the norms in which the solution is estimated before and after the transmission time $t_{2}$. This transmission condition turns out to be independent of whether a strong or weak imposition is used. We will also discuss the implications of weighted norms on the transmission conditions and energy estimates.

Semi-discrete and fully discrete transmission problems will also be considered. In the latter cases, we utilise the theory of Summation-by-Parts (SBP) operators, first introduced in $[5,6]$ in order to provide a means of obtaining stable finite difference procedures for the spatial discretisation of initial-boundary value problems. Since then, the SBP framework has been extended to methods outside the finite difference paradigm, including finite volume methods [7, 8, 9], spectral collocation, Galerkin and element methods [10, 11, 12], correction procedures via reconstruction [13] and temporal discretisations $[14,15,16,17]$. This opens the door to utilising energy arguments in order to analyse the stability of fully discrete numerical schemes, if initial, boundary and transmission conditions are imposed weakly [18, 19].

It will be shown that all fully discrete transmission problems considered must satisfy a certain condition in order to obtain an energy estimate. This condition is completely analogous to the one obtained in the continuous setting. Throughout the paper we aim to keep the governing equations - continuous or discrete - as general as possible. Thus, the conditions derived will apply to a wide range of problems and numerical schemes, and may serve as an a priori test for the availability of an energy estimate in a given norm.

The remainder of the paper is structured as follows: In section 2 we introduce the notation and definitions required henceforth. The transmission problem is formally introduced in section 3 and a necessary and sufficient condition for energy boundedness is derived for both strong and weak formulations. In section 4 , a discrete transmission problem is presented, and a stability analysis using a weak formulation is performed. We return to the transmission condition in section 5, show how to find weighted norms such that it is satisfied, and discuss its implications. A selection of appli- 
cations illustrating the preceding theory are presented in section 6. Finally, conclusions are drawn in section 7 .

\section{Preliminaries}

Before proceeding we introduce the necessary notation and definitions.

\subsection{Semi-boundedness and well-posedness}

Consider the initial-boundary value problem (IBVP)

$$
\begin{aligned}
& u_{t}+\mathcal{D}(u)=F, \quad t>0, \quad x \in \Omega, \\
& \mathcal{B}(u)=g, \quad t \geq 0, \quad x \in \Gamma, \\
& u=f, \quad t=0, \quad x \in \Omega \cup \Gamma,
\end{aligned}
$$

where $\Omega$ is an open $d$-dimensional region and $\mathcal{D}$ is a differential operator. The operator $\mathcal{B}$ defines a set of boundary conditions on the boundary $\Gamma$ of $\Omega$, and the functions $F, g$ and $f$ are given forcing, boundary and initial data.

For two functions $u$ and $v$ defined on $\Omega$, we introduce the inner product and norm

$$
(u, v)_{P}=\int_{\Omega} u^{\top} P v \mathrm{~d} x, \quad\|u\|_{P}=(u, u)_{P}^{1 / 2}
$$

where $P$ is a positive definite matrix whose dimension matches the number of variables contained in the vectors $u$ and $v$.

Definition 1. Let $\mathbb{V}$ be the space of differentiable functions satisfying the boundary conditions $\mathcal{B}(v)=0$. The differential operator $\mathcal{D}$ is semi-bounded if for all $v \in \mathbb{V}, \mathcal{D}(v)$ satisfies the inequality

$$
(v, \mathcal{D}(v))_{P} \geq 0 .
$$

Definition 2. The differential operator $\mathcal{D}$ is maximally semi-bounded if it is semi-bounded in the function space $\mathbb{V}$ but not in any space with fewer boundary conditions.

Note that if $\mathcal{D}$ is semi-bounded (or maximally semi-bounded) in (1), and $F=g=0$, then we can estimate the solution $u$ since

$$
\frac{\mathrm{d}}{\mathrm{d} t}\|u\|_{P}^{2}=2\left(u, u_{t}\right)_{P}=-2(u, \mathcal{D}(u))_{P} \leq 0,
$$

which after integration gives

$$
\|u(x, t)\|_{P} \leq\|f(x)\|_{P} .
$$


Definition 3. The IBVP (1) with $F=g=0$ is well-posed if for every sufficiently smooth $f$ compatible with the boundary data, and every finite time interval $0 \leq t \leq \tau$, it has a unique smooth solution satisfying the estimate

$$
\|u\|_{P} \leq K_{c} e^{\alpha_{c} t}\|f\|_{P}, \quad 0 \leq t \leq \tau
$$

where $K_{c}$ and $\alpha_{c}$ are constants independent of $f$.

Comparing (4) and (5) it is clear that if $\mathcal{D}$ in (1) is maximally semibounded, and hence a solution exists, well-posedness follows. In general terms, we say that the solution $u$ satisfies an energy estimate if it is bounded in terms of data such as in (5), or in some other way.

Remark 1. The condition for semi-boundedness in Definition 1 may be relaxed to $(v, \mathcal{D}(v))_{P} \geq-\alpha\|v\|_{P}^{2}$ for non-zero data $F$ and $g$. However, there is no chance of obtaining a semi-bounded problem for non-zero data with $\alpha>0$ (disregarding zero order terms) unless one is already available for zero data with $\alpha=0$ [1, 2, 20]. We therefore adopt Definition 1 in the remainder.

Remark 2. Henceforth, we will be concerned with the coupling of problems of the form (1) at some given time $t=t_{2}$. Well-posedness of such couplings are independent of $F$ and $g$, whence for notational brevity, we set $F=g=0$ in the remainder. We also assume that the boundary operator $\mathcal{B}$ contains a minimal set of boundary conditions such that $\mathcal{D}$ is maximally semi-bounded.

Remark 3. The results in this paper are valid also for non-linear operators $\mathcal{D}(v)$ satisfying condition (3) (the coupling is linear in time). However, for simplicity of presentation, we discuss the problem in the linear setting.

\subsection{Strong and weak formulations}

Throughout the paper, an IBVP formulated as (1) indicates a strong imposition of the boundary conditions, where the operator $\mathcal{B}$ is defined only on the domain boundary $\Gamma$. However, we may also define $\mathcal{B}$ on the whole domain and consider weak formulations of the form

$$
\begin{aligned}
& u_{t}+\mathcal{D}(u)=\mathcal{L}\left(\Sigma_{\Gamma} \mathcal{B}(u)\right), \quad t>0, \quad x \in \Omega \cup \Gamma, \\
& u=f, \quad t=0, \quad x \in \Omega \cup \Gamma \text {. }
\end{aligned}
$$

Here, $\mathcal{L}$ is a lifting operator $[3,4]$, which imposes the boundary conditions in a weak sense, and is defined through the relation

$$
(u, \mathcal{L}(v))_{P}=\oint_{\Gamma} u^{\top} v \mathrm{~d} s
$$


The penalty matrix $\Sigma_{\Gamma}$ in (6) will be chosen in order to obtain an energybound.

We will sometimes abbreviate the operator $\mathcal{D}(u)-\mathcal{L}\left(\Sigma_{\Gamma} \mathcal{B}(u)\right)$ with $\mathcal{D}_{\mathcal{L}}(u)$. When handling weak boundary conditions, maximal semi-boundedness will refer to $\mathcal{D}_{\mathcal{L}}(u)$ rather than $\mathcal{D}(u)$. The energy method applied to (1) or (6) is

$$
\frac{\partial}{\partial t}\|u\|_{P}^{2}=\left(u, u_{t}\right)_{P}+\left(u_{t}, u\right)_{P}=-(u, \mathcal{D})-(\mathcal{D}, u),
$$

followed by integration by parts. Here, $\mathcal{D}$ refers to either $\mathcal{D}(u)$ (strong formulation) or $\mathcal{D}_{\mathcal{L}}(u)$. The integration by parts procedure produces certain boundary terms. If they are negative semi-definite, $\mathcal{D}$ is maximally semibounded and well-posedness follows. If they are positive, there is in general no way of estimating them in terms of $\|u\|_{P}$ and obtaining well-posedness.

The initial condition may be imposed weakly in the same way as the boundary conditions, by adding another lifting operator to (6). Details of such a problem will be presented in section 3.2.

\subsection{Semi-discrete stability}

A semi-discretisation of (1) or (6) with $F=g=0$ is given by

$$
\begin{aligned}
\mathbf{u}_{t}+D(\mathbf{u}) & =0, \quad t>0 \\
\mathbf{u} & =\mathbf{f}, \quad t=0
\end{aligned}
$$

where $\mathbf{u}^{\top}=\left(\mathbf{u}_{1}^{\top}, \ldots, \mathbf{u}_{d}^{\top}\right)$ with $\mathbf{u}_{j}^{\top}=\left(u_{0}^{(j)}, \ldots, u_{n_{j}}^{(j)}\right), j=1, \ldots, d$ is a grid vector approximating the function $u(x, t)$ on the $d$-dimensional grid $\mathbf{x}^{\top}=$ $\left(\mathbf{x}_{1}^{\top}, \ldots, \mathbf{x}_{d}^{\top}\right)$. Here, $\mathbf{x}_{j}^{\top}=\left(x_{0}^{(j)}, \ldots, x_{n_{j}}^{(j)}\right)$ and $x_{k}^{(j)}=x_{0}^{(j)}+\sum_{l=1}^{k} h_{l}^{(j)}$, where $h_{l}^{(j)}$ denotes local step sizes in the $j$ th spatial dimension.

The spatial discretisation $D(\mathbf{u})$ approximates the differential operator $\mathcal{D}$ augmented with the boundary operator $\mathcal{B}$. The grid vector $\mathbf{f}$ is obtained by projecting the data $f$ onto the spatial grid.

We introduce the discrete inner product and norm

$$
(\mathbf{u}, \mathbf{v})_{h}=\mathbf{u}^{\top} P_{\mathbf{x}} \mathbf{v}, \quad\|\mathbf{u}\|_{h}=(\mathbf{u}, \mathbf{u})_{h}^{1 / 2}
$$

where the positive definite matrix $P_{\mathbf{x}}$ is such that (8) approximates the continuous inner product and norm in (2) on the grid $\mathbf{x}$. 
Definition 4. The semi-discretisation (7) is stable if for every sufficiently smooth grid vector $\mathbf{f}$, and for every finite time interval $0 \leq t \leq \tau$, the estimate

$$
\|\mathbf{u}\|_{h} \leq K_{d} e^{\alpha_{d} t}\|\mathbf{f}\|_{h}, \quad 0 \leq t \leq \tau
$$

holds for all sufficiently fine grids. The constants $K_{d}$ and $\alpha_{d}$ are independent of $\mathbf{f}$ and the grid.

Here, smooth grid vector refers to the projection of a smooth function onto the grid. Note that if $D(\mathbf{u})$ in (7) is semi-bounded in the inner product (8), an energy estimate and stability follows in the same way as for the maximally semi-bounded continuous problem.

Remark 4. Since existence is not an issue for discrete problems (consistency and stability suffice), semi-boundedness is the relevant concept.

\subsection{Fully discrete stability and SBP in time}

An SBP operator may be defined as follows:

Definition 5. A matrix $D=P^{-1} Q$ is an $S B P$ operator of order $q$ if

1. $D \mathbf{x}^{m}=m \mathbf{x}^{m-1}, \quad m=0, \ldots, q$,

2. $P=P^{T}>0$,

3. $Q+Q^{\top}=\operatorname{diag}(-1,0, \ldots, 0,1)$.

Recently, the SBP framework was extended to more general approximations $[10,21]$ as well as temporal discretisation [14]. As an example of the temporal procedure, consider as a special case of (1), the initial-value problem

$$
\begin{array}{rlrl}
u_{t}+\lambda u & =0, & 0<t<T, \\
u & =f, & & t=0,
\end{array}
$$

where $\lambda$ is a complex constant with $\operatorname{Re}(\lambda) \geq 0$.

The energy method applied to (10) (multiplying by the complex conjugated solution $\bar{u}$ and integrating in time) leads to the bound

$$
u^{2}(T)+2 \operatorname{Re}(\lambda)\|u\|^{2}=f^{2},
$$

where $\|u\|^{2}=\int_{0}^{T}|u|^{2} \mathrm{~d} t$.

Applying SBP in time to (10) yields

$$
P^{-1} Q \mathbf{u}+\lambda \mathbf{u}=\sigma P^{-1}\left(u_{0}-f\right) \mathbf{e}_{0},
$$


where $\mathbf{e}_{0}=(1,0, \ldots, 0)^{\top}$ and $\sigma$ is a scalar penalty parameter yet to be determined. Here, $\mathbf{u}$ approximates the continuous solution $u$ at each grid point in time. The right-hand side contains the Simultaneous Approximation Term (SAT) [18] that weakly imposes the initial condition. The SAT term is an example of a discrete lifting operator.

Choosing $\sigma=-1$ and applying the discrete energy method to (12) (multiplying from the left by $\mathbf{u}^{*} P$, adding the conjugate transpose and using Definition 5) leads to

$$
\left|u_{n}\right|^{2}+2 \lambda\|\mathbf{u}\|_{h}^{2}=|f|^{2}-\left|u_{0}-f\right|^{2} .
$$

In (13), $\|\mathbf{u}\|_{h}^{2}=\mathbf{u}^{*} P \mathbf{u}$, where $\mathbf{u}^{*}$ denotes the conjugate transpose of the vector u. Note that (13) mimics (11) up to the small dissipative term $\left|u_{0}-f\right|^{2}$, which vanishes with grid refinements.

Definition 6. A full discretisation of the IBVP (1) or (6) is stable if the estimate (9) holds at the final time.

Clearly, (13) implies $\left|u_{n}\right|^{2} \leq|f|^{2}$, and hence the SBP discretisation (12) is stable. For further reading about SBP in time, see [15, 16, 17]. For comprehensive reviews of SBP-SAT and examples of its use, see [22, 23].

\section{Well-posedness of transmission problems}

In this section we formally define the transmission problem and derive a necessary and sufficient condition for an energy estimate.

\subsection{The strong formulation}

Consider the following general coupled model problem:

$$
\begin{aligned}
& u_{t}+\mathcal{D}_{1}(u)=0, \quad t_{1}<t<t_{2}, \quad x \in \Omega, \\
& v_{t}+\mathcal{D}_{2}(v)=0, \quad t_{2}<t<t_{3}, \quad x \in \Omega, \\
& \mathcal{B}_{1}(u)=0, \quad t_{1} \leq t \leq t_{2}, \quad x \in \Gamma, \\
& \mathcal{B}_{2}(v)=0, \quad t_{2} \leq t \leq t_{3}, \quad x \in \Gamma, \\
& u=f_{1}, \quad t=t_{1}, \quad x \in \Omega \cup \Gamma, \\
& v=\mathcal{X}(u), \quad t=t_{2}, \quad x \in \Omega \cup \Gamma \text {. }
\end{aligned}
$$

We assume that (14) offers a complete and well-posed description of the underlying dynamics. By this we mean that there are positive definite matrices 
$P_{1,2}$ such that the operators $\mathcal{D}_{1,2}$ are maximally semi-bounded in the inner products induced by $P_{1,2}$. Hence, in the case $\mathcal{X}(u)=f_{2}$, where $f_{2}$ is solutionindependent data, (14) is well-posed in the sense of Definition 3.

We refer to (14) as the strong transmission problem. This terminology is motivated by the fact that (14) describes any scenario where the dynamics governing the solution $u$ is interrupted at time $t=t_{1} ; u$ is subject of the operation $\mathcal{X}$; and the resulting information is transmitted to $v$, after which the governing dynamics may have changed.

Note that the operator $\mathcal{X}$ accepts the vector argument $u$ and must return a vector of the same dimension as $v$ for (14) to make sense. We will generally let $\mathcal{X}$ admit a matrix representation $X$, such that we may write

$$
\mathcal{X}(u)=X u \text {. }
$$

Of course, $X$ may still implicitly depend on $u$ through its matrix elements.

Our goal is to investigate when (14) is well-posed, and in particular when sharp bounds can be obtained. The energy method gives $\left(u, u_{t}\right)_{P_{1}} \leq 0$ and $\left(v, v_{t}\right)_{P_{2}} \leq 0$. Summing the two inequalities and integrating in time yields

$$
\left\|v\left(x, t_{3}\right)\right\|_{P_{2}}^{2} \leq\left\|f_{1}(x)\right\|_{P_{1}}^{2}-\left.\int_{\Omega} u^{\top}\left\{P_{1}-X^{\top} P_{2} X\right\} u \mathrm{~d} x\right|_{t=t_{2}} .
$$

From (15) we immediately have

Proposition 1. An energy estimate can be obtained for the strong transmission problem (14) if and only if the transmission condition

$$
P_{1}-X^{\top} P_{2} X \geq 0
$$

is satisfied at time $t=t_{2}$.

Before proceeding it is appropriate to remark that by assumption, $\mathcal{D}_{1}(u)$ is maximally semi-bounded, whence $u$ satisfies the estimate

$$
\left\|u\left(x, t_{2}\right)\right\|_{P_{1}}^{2} \leq\left\|f_{1}(x)\right\|_{P_{1}}^{2} .
$$

Consequently we could define new data $f_{2}=\left.X u\right|_{t=t_{2}}$ and treat the problem for $v$ in $(14)$ as a stand-alone IBVP. Since $\mathcal{D}_{2}(v)$ is also maximally semibounded, $v$ would satisfy the estimate

$$
\left\|v\left(x, t_{3}\right)\right\|_{P_{2}}^{2} \leq\left\|f_{2}(x)\right\|_{P_{2}}^{2},
$$


ultimately yielding a non-sharp estimate for $\left\|v\left(x, t_{3}\right)\right\|_{P_{2}}$.

However, there are several reasons for why this approach is undesirable: Firstly, $f_{2}$ is not available a priori and hence it is unknown whether $\left\|f_{2}\right\|_{P_{2}}$ is large. This is particularly evident for problems with non-zero boundary data $g$ and forcing $F$, where $u$ will satisfy an estimate [1] of the form

$$
\left\|u\left(x, t_{2}\right)\right\|_{P_{1}}^{2} \leq K e^{\alpha\left(t_{2}-t_{1}\right)}\left(\left\|f_{1}(x)\right\|_{P_{1}}^{2}+\int_{t_{1}}^{t_{2}}\left(\|F\|_{\tilde{P}_{1}}^{2}+\|g\|_{\Gamma}^{2}\right) \mathrm{d} t\right) .
$$

In (18), $\|\cdot\|_{\Gamma}$ is a norm defined on the boundary of the spatial domain. Thus, $\left\|u\left(x, t_{2}\right)\right\|_{P_{1}}$ may be large compared to $\left\|f_{1}\right\|_{P_{1}}$.

Note also that from definitions 3,4 and 6 of well-posedness and stability that the estimates of the solutions are formulated in terms of initially available data. Hence, we only label estimates that are obtained in terms of $f_{1}$ as energy estimates in the remainder of this paper.

Furthermore, introducing $f_{2}$ at time $t_{2}$ enforces $u$ and $v$ to be obtained sequentially from (14). This property is inherited by any discretisation of (14), which renders parallel implementations impossible. Thus, even if it is possible to compute a numerical solution using $f_{2}$, the effect on the efficiency would be detrimental. In light of these considerations, we will not discuss the above formally uncoupled approach further.

\subsection{The weak formulation}

A weak formulation of (14) is given by

$$
\begin{array}{lll}
u_{t}+\mathcal{D}_{\mathcal{L}_{1}}(u)=\mathcal{L}_{t_{1}}\left(\Sigma_{t_{1}}\left(u-f_{1}\right)\right), & t_{1} \leq t<t_{2}, & x \in \Omega \cup \Gamma, \\
v_{t}+\mathcal{D}_{\mathcal{L}_{2}}(v)=\mathcal{L}_{t_{2}}\left(\Sigma_{t_{2}}(v-X u)\right), & t_{2} \leq t<t_{3}, & x \in \Omega \cup \Gamma .
\end{array}
$$

We refer to (19) as the weak transmission problem. In the event that $\mathcal{X}(u)=$ $X u=f_{2}$ is solution-independent data, we assume that (19) forms a wellposed problem. The lifting operators $\mathcal{L}_{t_{1}}$ and $\mathcal{L}_{t_{2}}$ impose the initial and transmission conditions weakly and are defined through the relations

$$
\int_{t_{1}}^{t_{2}}\left(u, \mathcal{L}_{t_{1,2}}(v)\right)_{P_{1,2}} \mathrm{~d} t=\left.(u, v)_{P_{1,2}}\right|_{t=t_{1,2}} .
$$

Our goal is to find the conditions under which there exists a penalty matrix $\Sigma_{t_{2}}$ such that the solution $v$ of (19) satisfies an energy estimate. 
The energy method applied to (19), followed by integration in time, gives

$$
\begin{aligned}
& \left\|u\left(x, t_{2}\right)\right\|_{P_{1}}^{2} \leq\left\{\|u\|_{P_{1}}^{2}+\left(u, \Sigma_{t_{1}}\left(u-f_{1}\right)\right)_{P_{1}}+\left(\Sigma_{t_{1}}\left(u-f_{1}\right), u\right)_{P_{1}}\right\}_{t=t_{1}} \\
& \left\|v\left(x, t_{3}\right)\right\|_{P_{2}}^{2} \leq\left\{\|v\|_{P_{2}}^{2}+\left(v, \Sigma_{t_{2}}(v-X u)\right)_{P_{2}}+\left(\Sigma_{t_{2}}(v-X u), v\right)_{P_{2}}\right\}_{t=t_{2}} .
\end{aligned}
$$

To bound the terms evaluated at $t=t_{1}$ in (20), we choose $\Sigma_{t_{1}}=-I_{1}$, where $I_{1}$ is the identity matrix of the same dimension as $P_{1}$. Adding and subtracting $\left\|f_{1}\right\|_{P_{1}}^{2}$ to these terms gives

$$
\begin{aligned}
\left\{\|u\|_{P_{1}}^{2}\right. & \left.-\left(u, u-f_{1}\right)_{P_{1}}-\left(u-f_{1}, u\right)_{P_{1}} \pm\left\|f_{1}\right\|_{P_{1}}^{2}\right\}_{t=t_{1}} \\
& =\int_{\Omega}\left\{f_{1}^{\top} P_{1} f_{1}-\left(u-f_{1}\right)^{\top} P_{1}\left(u-f_{1}\right)\right\}_{t=t_{1}} \mathrm{~d} x \\
& =\left\{\left\|f_{1}\right\|_{P_{1}}^{2}-\left\|\left(u-f_{1}\right)\right\|_{P_{1}}^{2}\right\}_{t=t_{1}},
\end{aligned}
$$

which clearly is bounded.

Inserting (21) into (20) and adding the two inequalities results in

$$
\begin{aligned}
\left\|v\left(x, t_{3}\right)\right\|_{P_{2}}^{2} & \leq\left\{\left\|f_{1}\right\|_{P_{1}}^{2}-\left\|\left(u-f_{1}\right)\right\|_{P_{1}}^{2}\right\}_{t=t_{1}}+\left\{-\|u\|_{P_{1}}^{2}+\|v\|_{P_{2}}^{2}\right. \\
& \left.+\left(v, \Sigma_{t_{2}}(v-X u)\right)_{P_{2}}+\left(\Sigma_{t_{2}}(v-X u)\right)_{P_{2}}, v\right\}_{t=t_{2}} .
\end{aligned}
$$

In order to obtain an energy estimate for $v$, we must find a penalty matrix $\Sigma_{t_{2}}$ such that the terms evaluated at time $t=t_{2}$ in (22) are negative semidefinite. The following proposition states when such a matrix can be found.

Proposition 2. An energy estimate can be obtained for the weak transmission problem (19) if and only if the transmission condition (16) is satisfied at time $t=t_{2}$.

Proof. Let $T$ denote the terms in (22) that are evaluated at time $t=t_{2}$. Then $T$ may be written

$$
T=\left(\begin{array}{l}
u \\
v
\end{array}\right)^{\top} \underbrace{\left(\begin{array}{cc}
-P_{1} & -\left(P_{2} \Sigma_{t_{2}} X\right)^{\top} \\
-\left(P_{2} \Sigma_{t_{2}} X\right) & \left(P_{2} \Sigma_{t_{2}}\right)+\left(P_{2} \Sigma_{t_{2}}\right)^{\top}+P_{2}
\end{array}\right)}_{M}\left(\begin{array}{l}
u \\
v
\end{array}\right) .
$$

We begin by proving that (16) is a necessary condition. Thus, assume that $T \leq 0$, which implies that the symmetric matrix $M$ is negative semi-definite. Let $S=\left(I_{1}, X^{\top}\right)$. By Sylvester's theorem,

$$
S M S^{\top}=-P_{1}+X^{\top} P_{2} X \leq 0
$$


must hold, which is equivalent to (16); hence the condition is necessary.

Next, we show that (16) is a sufficient condition. Assume that (16) is satisfied. We add and subtract $(X u)^{\top} P_{2}(X u)$ from $T$ and choose $\Sigma_{t_{2}}=-I_{2}$, where $I_{2}$ is the identity matrix of the same dimension as $P_{2}$, to obtain

$$
T=\left(\begin{array}{c}
X u \\
v
\end{array}\right)^{\top}\left(\begin{array}{cc}
-P_{2} & P_{2} \\
P_{2} & -P_{2}
\end{array}\right)\left(\begin{array}{c}
X u \\
v
\end{array}\right)-u^{\top}\left(P_{1}-X^{T} P_{2} X\right) u .
$$

The rightmost term is negative semi-definite by condition (16). The matrix in the left term can be written

$$
\left(\begin{array}{cc}
-P_{2} & P_{2} \\
P_{2} & -P_{2}
\end{array}\right)=\left(\begin{array}{cc}
-1 & 1 \\
1 & -1
\end{array}\right) \otimes P_{2} \equiv B \otimes P_{2}
$$

where $\otimes$ denotes the Kronecker product. Since $P_{2}$ is positive definite and $B$ has eigenvalues $\{0,-2\}, T$ is negative semi-definite. Hence (22) is bounded by data and an energy estimate for (19) is obtained.

Remark 5. The proof of Proposition 2 shows that if (16) is satisfied, the choice $\Sigma_{t_{2}}=-I_{2}$ leads to an energy estimate. However, other choices of $\Sigma_{t_{2}}$ are also possible.

If condition (16) is satisfied, then the strong and weak implementation yields the same energy estimate, $\|v\|_{P_{2}} \leq\left\|f_{1}\right\|_{P_{1}}$, since $\left\|u\left(x, t_{1}\right)-f_{1}\right\|_{P_{1}}^{2}=0$ in (22). Thus, (16) is a completely general condition that must be satisfied independent of the way in which the transmission condition is implemented.

\section{Stability of discrete transmission problems}

Energy estimates for semi-discrete transmission problems is essentially covered by the analysis presented in section 3 . All that remains is to replace $\mathcal{D}_{1,2}$ by $D_{1,2}, u$ and $v$ by $\mathbf{u}$ and $\mathbf{v}$, and $f$ by $\mathbf{f}$ as appropriate. We therefore immediately have

Proposition 3. An energy estimate can be obtained for the semi-discrete transmission problems (14) and (19) if and only if the transmission condition (16) is satisfied at time $t=t_{2}$. 
Next, we focus our attention on fully discrete schemes where the temporal discretisation is performed using SBP in time. The corresponding transmission problem is given by

$$
\begin{aligned}
\left(P_{t,(u)}^{-1} Q_{t,(u)} \otimes I_{x,(u)}\right) \mathbf{u} & +I_{t,(u)} \otimes D_{1}(\mathbf{u}) \\
& =\left(P_{t,(u)}^{-1} E_{0,(u)} \otimes \Sigma_{h, t_{1}}\right)\left(I_{t,(u)} \otimes\left(\mathbf{u}_{0}-\mathbf{f}_{1}\right)\right), \\
\left(P_{t,(v)}^{-1} Q_{t,(v)} \otimes I_{x,(v)}\right) \mathbf{v} & +I_{t,(v)} \otimes D_{2}(\mathbf{v}) \\
& =\left(P_{t,(v)}^{-1} E_{0,(v)} \otimes \Sigma_{h, t_{2}}\right)\left(I_{t,(v)} \otimes\left(\mathbf{v}_{0}-X \mathbf{u}_{n}\right)\right) .
\end{aligned}
$$

With a slight abuse of notation, $\mathbf{u}$ and $\mathbf{v}$ denote grid vectors on a $d+1$ dimensional spatio-temporal grid in a similar fashion to (7). We assume that $D_{1}(\mathbf{u})$ and $D_{2}(\mathbf{v})$ are semi-bounded in inner products induced by the symmetric positive definite matrices $P_{x,(u, v)}$, such that when $X \mathbf{u}_{n}=\mathbf{f}_{2}$ is solution-independent data, (23) is stable in the sense of Definition 6 .

In (23), $I$ and $E$ (with appropriate subscripts) respectively denote the identity matrix and the square matrix whose elements are one in the top-left corner and zero elsewhere. The right-hand sides contain SAT penalty terms, i.e. discrete lifting operators that weakly enforce the initial and transmission conditions in a way analogous to the right-hand sides of (19). The vectors $\mathbf{u}_{n}$ and $\mathbf{v}_{0}$ respectively denote the numerical solution at time $t_{2}$ before and after application of $X$. Finally, $\Sigma_{h, t_{1}}$ and $\Sigma_{h, t_{2}}$ are penalty matrices yet to be determined.

Multiplying (23) from the left by $\mathbf{u}^{\top}\left(P_{t,(u)} \otimes P_{x,(u)}\right)$ and $\mathbf{v}^{\top}\left(P_{t,(v)} \otimes P_{x,(v)}\right)$ as appropriate, adding the transpose of the result, applying Definition 5 and adding the two equations, leads to

$$
\begin{aligned}
\left\|\mathbf{v}_{m}\right\|_{P_{x,(v)}}^{2} & =\left\|\mathbf{f}_{1}\right\|_{P_{x,(u)}}^{2}-\left\|\mathbf{u}_{0}-\mathbf{f}_{1}\right\|_{P_{x,(u)}}^{2}-\left\|\mathbf{u}_{n}\right\|_{P_{x,(u)}}^{2}+\left\|\mathbf{v}_{0}\right\|_{P_{x,(v)}}^{2} \\
& +\left(\mathbf{v}_{0}, \Sigma_{h, t_{2}}\left(\mathbf{v}_{0}-X \mathbf{u}_{n}\right)\right)_{P_{x,(v)}}+\left(\Sigma_{h, t_{2}}\left(\mathbf{v}_{0}-X \mathbf{u}_{n}\right), \mathbf{v}_{0}\right)_{P_{x,(v)}}
\end{aligned}
$$

Here we have used $\Sigma_{h, t_{1}}=-I_{x,(u)}$ and performed a calculation similar to the one in (21) in order to obtain the first two terms.

For (23) to be energy stable, we need the right-hand side of (24) to be bounded. This requires finding a penalty matrix $\Sigma_{h, t_{2}}$ such that the transmission terms involving $\mathbf{u}_{n}$ and $\mathbf{v}_{0}$ are negative semidefinite. The following proposition establishes the conditions under which this is possible: 
Proposition 4. A penalty matrix $\Sigma_{h, t_{2}}$ exists such that (23) is stable if and only if the transmission condition (16) holds with $P_{1}=P_{x,(u)}$ and $P_{2}=P_{x,(v)}$.

Proof. The estimate (24) is term for term analogous to the continuous energy estimate (22). The proof is therefore identical to that of Proposition 2.

\section{Scaled norms}

The transmission condition (16) is completely general and applies to any problem of the form (14), (19) or (23). In this section we show that norminducing matrices $P_{1,2}$ may always be found such that (16) is satisfied. We also discuss the implications of this fact.

Let $\kappa>0$ be a real constant. Our starting point is the observation that if the solution to an IBVP satisfies an energy estimate in the norm $\|\cdot\|_{P}$, then it also satisfies an estimate in the scaled norm $\|\cdot\|_{\kappa P} \equiv \sqrt{\kappa}\|\cdot\|_{P}$, which is clear from (5). We may thus redo the energy analysis for the strong transmission problem (14) using the scaled norm $\|u\|_{\kappa P_{1}}$ to obtain the energy rate

$$
\left\|v\left(x, t_{3}\right)\right\|_{P_{2}}^{2} \leq \kappa\left\|f_{1}(x)\right\|_{P_{1}}^{2}-\left.\int_{\Omega} u^{\top}\left\{\kappa P_{1}-X^{\top} P_{2} X\right\} u \mathrm{~d} x\right|_{t=t_{2}} .
$$

Analogous energy rates hold for the weak and discrete transmission problems. Clearly, the transmission condition (16) is now replaced by the scaled transmission condition

$$
\kappa P_{1}-X^{\top} P_{2} X \geq 0 .
$$

Next, we investigate if we can find a $\kappa$ such that (26) is satisfied.

Let $\lambda_{\max }(H)=\max _{j \in\{1, \ldots, n\}}\left|\lambda_{j}(H)\right|$ denote the spectral radius of an $n \times n$ matrix $H$. Let $\lambda_{\min }(H)$ be defined similarly.

Proposition 5. The scaled transmission condition (26) is satisfied if

$$
\kappa \geq \frac{\lambda_{\max }\left(X^{\top} P_{2} X\right)}{\lambda_{\min }\left(P_{1}\right)}
$$

The proof is found in Appendix A. Proposition 5 reveals that as long as the governing equations for the solutions $u$ and $v$ satisfy energy estimates, then so does the corresponding transmission problem, if the norms $P_{1,2}$ are scaled appropriately. In other words, the temporal coupling of two well-posed (or stable) problems preserves the well-posedness (stability). 
However, there might be drawbacks with the scaling procedure described above. Replacing the transmission condition (16) with the scaled condition (26) changes the energy estimate (15) for the strong transmission problem to

$$
\left\|v\left(x, t_{3}\right)\right\|_{P_{2}}^{2} \leq\left\|f_{1}(x)\right\|_{\kappa P_{1}}^{2}=\kappa\left\|f_{1}(x)\right\|_{P_{1}}^{2} .
$$

Analogous results hold for the weak and discrete transmission problems. If $\kappa>1$, the scaled estimate (28) is clearly weaker than the unscaled estimate (15). In a problem involving $m$ transmissions, each requiring scaled norms satisfying

$$
\kappa_{j} P_{j}-X_{j}^{\top} P_{j+1} X_{j} \geq 0, \quad j=1, \ldots m,
$$

at time $t=t_{j+1}$, the final energy estimate becomes

$$
\left\|v\left(x, t_{m}\right)\right\|_{P_{m+1}}^{2} \leq\left\|f_{1}(x)\right\|_{P_{1}}^{2} \prod_{j=1}^{m} \kappa_{j}
$$

If each $\kappa_{j}>1$, the estimate (29) may of course be very weak. This is an obvious disadvantage in situations where $v(x, t)$ represents an error or a small disturbance, and hence it is generally desirable to choose $X$ such that $\kappa_{j}$ becomes as small as possible.

Remark 6. The choice of $\kappa$ in (27) is not minimal, however it is often simple to calculate. As the proof of Proposition 5 suggests, $\kappa=\lambda_{\max }(H)$, where $H$ is given in (A.1) in Appendix A, is an optimal choice. However, calculating $\lambda_{\max }(H)$ requires that a spectral decomposition of $P_{1}$ is available.

We make a separate note of the important special case where $P_{1}=P_{2}$. In this case, we have the following necessary condition:

Proposition 6. If $P_{1}=P_{2}$, a necessary condition for the scaled transmission condition (26) to be satisfied is that

$$
\kappa \geq \lambda_{\max }^{2}(X)
$$

The proof is found in Appendix B. Verifying (30) is clearly simpler than (27), however (30) is in general not sufficient. In section 6.2.2 we will consider a problem where the choice of $\kappa$ has a significant impact on the energy estimate. 


\section{Applications}

In this section we describe a selection of applications that can be modeled as transmission problems. In some of these, $X$ can systematically be constructed such that the transmission conditions (16) is satisfied, while in others this poses challenges. The examples are chosen such that a minimal number of assumptions on $\mathcal{D}_{1,2}$ or $D_{1,2}$ are made. In what follows, we let the penalty matrix for the initial data be $-I$ such that we do not have to consider the initial conditions further.

\subsection{Continuous transmission problems}

We start by considering problems in continuous time, and exemplify with a derivation of an energy estimate followed by a multi-grid application.

\subsubsection{Acoustics}

Consider the linearised one-dimensional Euler equations,

$$
\mathbf{u}_{t}+A \mathbf{u}_{x}=0, \quad A=\left(\begin{array}{ccc}
\bar{u} & \bar{\rho} & 0 \\
0 & \bar{u} & \frac{1}{\bar{\rho}} \\
0 & \gamma \bar{p} & \bar{u}
\end{array}\right)
$$

augmented with initial and boundary conditions. Here, the elements of the solution vector $\mathbf{u}=(\rho, u, p)^{\top}$ denote small perturbations of density, velocity and pressure around a constant background flow $(\bar{\rho}, \bar{u}, \bar{p})^{\top}$ and $\gamma$ is the ratio of specific heats.

Under the assumption of an irrotational flow, the Euler equations may be simplified to the acoustic wave equation,

$$
\mathbf{v}_{t}+B \mathbf{v}_{x}=0, \quad B=\left(\begin{array}{cc}
0 & \bar{c} \\
\bar{c} & 0
\end{array}\right)
$$

where $\mathbf{v}=(p / \bar{\rho}, \bar{c} v)^{\top}$ and $\bar{c}=\sqrt{\gamma \bar{p} / \bar{\rho}}$ is the speed of sound in the fluid.

Consider a situation where we solve (31) up to time $t=t_{2}$, after which we switch to solve the simpler problem (32). Such a situation may arise when computational resources are limited, and where it is known that the solution approaches an irrotational state at $t=t_{2}$.

It is reasonable to impose that the pressure and velocity should not change during the switch. This corresponds to the transmission condition

$$
\mathbf{v}=X \mathbf{u}, \quad X=\left(\begin{array}{ccc}
0 & 0 & \frac{1}{\bar{\rho}} \\
0 & \bar{c} & 0
\end{array}\right)
$$


It remains to find norms in which (31) and (32) can be estimated. If we can find a matrix $S$ such that upon multiplying (31) by $S$, the resulting system

$$
(S \mathbf{u})_{t}+S A S^{-1}(S \mathbf{u})_{x}=0
$$

is symmetric, then the corresponding matrix inducing the proper norm is $P=S^{\top} S$. An analogous argument holds for (32).

A matrix that symmetries the Euler equations is [24]

$$
S_{1}=\left(\begin{array}{ccc}
\frac{1}{\beta \bar{\rho}} & 0 & -\frac{1}{\beta \bar{\rho} \bar{c}^{2}} \\
0 & \frac{1}{2 \bar{c}} & \frac{1}{2 \bar{\rho} \bar{c}^{2}} \\
0 & -\frac{1}{2 \bar{c}} & \frac{1}{2 \bar{\rho} \bar{c}^{2}}
\end{array}\right) \Rightarrow P_{1}=\left(\begin{array}{ccc}
\frac{1}{(\beta \bar{\rho})^{2}} & 0 & -\frac{1}{(\beta \bar{\rho} \bar{c})^{2}} \\
0 & \frac{1}{2 \bar{c}^{2}} & 0 \\
-\frac{1}{(\beta \bar{\rho} \bar{c})^{2}} & 0 & \frac{2+\beta^{2}}{2\left(\beta \bar{\rho} \bar{c}^{2}\right)^{2}}
\end{array}\right)
$$

where $\beta=\sqrt{2(\gamma-1)}$. Similarly, for the acoustic wave equation we use

$$
S_{2}=\frac{1}{2 \bar{c}^{2}}\left(\begin{array}{cc}
-1 & 1 \\
1 & 1
\end{array}\right) \Rightarrow P_{2}=\frac{1}{2 \bar{c}^{4}}\left(\begin{array}{ll}
1 & 0 \\
0 & 1
\end{array}\right)
$$

Using the operator $X$ defined in (33), the transmission condition (16) becomes

$$
0 \leq P_{1}-X^{\top} P_{2} X=\left(\begin{array}{ccc}
\frac{1}{(\beta \bar{\rho})^{2}} & 0 & -\frac{1}{(\beta \bar{\rho} \bar{c})^{2}} \\
0 & 0 & 0 \\
-\frac{1}{(\beta \bar{\rho} \bar{c})^{2}} & 0 & \frac{1}{\left(\beta \bar{\rho} \bar{c}^{2}\right)^{2}}
\end{array}\right)
$$

The matrix on the right-hand side has the eigenvalues $\lambda_{1}=\lambda_{2}=0$ and $\lambda_{3}=\left(1+\bar{c}^{4}\right) /\left(\beta \bar{\rho} \bar{c}^{2}\right)^{2}>0$. Thus, the transmission condition (16) is satisfied and an energy estimate can be obtained.

\subsubsection{Multi-grid iterations}

We want to solve the following discretised boundary-value problem:

$$
D_{1} \mathbf{w}=\mathbf{F},
$$

augmented with suitable boundary conditions such that $D_{1}$ has eigenvalues with positive real parts. A possible approach is to introduce a pseudo-time derivative and solve the corresponding problem

$$
\begin{aligned}
\mathbf{u}_{t}+D_{1} \mathbf{u} & =\mathbf{F}, \quad t>0, \\
\mathbf{u} & =\mathbf{f}, \quad t=0,
\end{aligned}
$$

by marching in time until a steady state is reached. Here, $\mathbf{f}$ is arbitrary data. 
Convergence in (34) may be fast initially, but often stagnates after some time, say $t_{2}$. In order to accelerate the convergence we may use a multi-grid technique as follows: We introduce the residual $\mathbf{r}=D_{1}^{-1} \mathbf{F}-\mathbf{u}$ and define a residual equation

$$
D_{2} \mathbf{y}=I_{r} D_{1} \mathbf{r}
$$

where $D_{2}$ is a coarse grid operator obtained by restricting $D_{1}$ to a coarser mesh using the restriction operator $I_{r}$. Assuming that the residual equation can be solved exactly, we obtain $\mathbf{y}=D_{2}^{-1} I_{r}\left(\mathbf{F}-D_{1} \mathbf{u}\right)$.

At this stage, we return to the fine grid problem (34) by applying a prolongation operator $I_{p}$ to $\mathbf{y}$, and solve

$$
\begin{aligned}
\mathbf{v}_{t}+D_{1} \mathbf{v} & =\mathbf{F}, & & t>t_{2}, \\
\mathbf{v} & =\mathbf{u}+I_{p} D_{2}^{-1} I_{r}\left(\mathbf{F}-D_{1} \mathbf{u}\right), & & t=t_{2} .
\end{aligned}
$$

The above procedure may of course be performed in several cycles using multiple grid levels obtained by repeated applications of the restriction and prolongation operators.

If $\mathbf{F}=0$, then (34) and (35) combine to form the following semi-discrete transmission problem:

$$
\begin{aligned}
& \mathbf{u}_{t}+D_{1} \mathbf{u}=-\mathcal{L}_{t_{1}}\left(\mathbf{u}_{0}-\mathbf{f}\right) \\
& \mathbf{v}_{t}+D_{1} \mathbf{v}=\mathcal{L}_{t_{2}}\left(\Sigma_{t_{2}}\left(\mathbf{v}_{0}-\left(I_{x}-Y\right) \mathbf{u}_{n}\right)\right)
\end{aligned}
$$

where $Y=I_{p} D_{2}^{-1} I_{r} D_{1}$.

To obtain an energy estimate, condition (16) implies that we must have

$$
0 \leq P_{x}-\left(I_{x}-Y\right)^{\top} P_{x}\left(I_{x}-Y\right)
$$

for some positive definite matrix $P_{x}$. Since $Y$ is problem dependent, this condition has to be checked on a case to case basis. However, Proposition 6 gives the necessary condition $\kappa=1 \geq \lambda_{\max }^{2}\left(I_{x}-Y\right)$. Clearly this implies that the real part $\Re\left[\lambda_{j}\left(I_{x}-Y\right)\right] \leq 1$ for every $j$, from which it follows that

$$
\Re\left[\lambda_{j}(Y)\right] \geq 0, \quad \forall j .
$$

Consequently, (37) is necessary in order to obtain an energy estimate for the implementation (36).

Remark 7. If the so called Galerkin condition $D_{2}=I_{r} D_{1} I_{p}$ is satisfied it can be shown [25] that $Y$ has eigenvalues equal to 0 or 1, and hence (37) is satisfied. For details about multi-grid methods in the SBP setting, see [26]. 


\subsection{Discrete transmission problems}

Next, we consider problems in discrete time, and exemplify with interpolation in multi-block couplings and a filtering procedure.

\subsubsection{Temporal interpolation between time-blocks}

Interpolation between computational domains is a frequently arising problem that has attracted much attention. A range of methods exist that use overlapping grids [27], introduce ghost points [28], or recently, weakly impose continuity across domain interfaces using SAT techniques [29, 30]. Though the literature on the subject is largely concerned with interpolation between spatial domains, the same principle may be applied in time.

In [29], so called SBP preserving interpolation operators were introduced that successfully achieve stable and accurate spatial interpolation. Let $I_{F 2 C}$ and $I_{C 2 F}$ respectively denote interpolation operators from a finer to a coarser grid, and vice versa. Then, the interpolation operators are said to be SBP preserving if the following property holds:

$$
P_{C} I_{F 2 C}=I_{C 2 F}^{\top} P_{F}
$$

Here, $P_{C}$ and $P_{F}$ are norm-inducing matrices defined on the coarse and fine grid respectively. Further, for hyperbolic or parabolic problems with characteristic interface conditions, it was shown in [29] that the following conditions must be satisfied together with (38) for stability:

$$
P_{C}\left(I_{C}-I_{F 2 C} I_{C 2 F}\right) \geq 0, \quad P_{F}\left(I_{F}-I_{C 2 F} I_{F 2 C}\right) \geq 0 .
$$

In (39), $I_{C}$ and $I_{F}$ are identity matrices on the coarse and fine grids respectively.

Let $\mathbf{u}$ be a discrete solution vector defined on a fine temporal grid and let $\mathbf{v}$ similarly be defined on a coarser grid. The transmission problem corresponding to interpolation between the grids is

$$
\begin{aligned}
\left(P_{t,(u)}^{-1} Q_{t,(u)} \otimes I_{x,(u)}\right) \mathbf{u} & +I_{t} \otimes D_{1}(\mathbf{u}) \\
= & -\left(P_{t,(u)}^{-1} E_{0,(u)} \otimes I_{x,(u)}\right)\left(I_{t,(u)} \otimes\left(\mathbf{u}_{0}-\mathbf{f}\right)\right), \\
\left(P_{t,(v)}^{-1} Q_{t,(v)} \otimes I_{x,(v)}\right) \mathbf{v} & +I_{t} \otimes D_{2}(\mathbf{v}) \\
= & \left(P_{t,(v)}^{-1} E_{0,(v)} \otimes \Sigma_{h, t_{2}}\right)\left(I_{t,(v)} \otimes\left(\mathbf{v}_{0}-I_{F 2 C} \mathbf{u}_{n}\right)\right),
\end{aligned}
$$


and the transmission condition (16) becomes

$$
P_{x,(u)}-I_{F 2 C}^{\top} P_{x,(v)} I_{F 2 C} \geq 0 .
$$

Observe from (38) that $I_{C 2 F}=P_{x,(u)}^{-1} I_{F 2 C}^{\top} P_{x,(v)}$. Then, (41) can be rewritten

$$
\begin{aligned}
0 \leq P_{x,(u)}-I_{F 2 C}^{\top} P_{x,(v)} I_{F 2 C} & =P_{x,(u)}\left(I_{x,(u)}-P_{x,(u)}^{-1} I_{F 2 C}^{\top} P_{x,(v)} I_{F 2 C}\right) \\
& =P_{x,(u)}\left(I_{x,(u)}-I_{C 2 F} I_{F 2 C}\right),
\end{aligned}
$$

which is precisely the second condition in (39). In the converse case, where we interpolate from a coarse to a fine grid, an analogous equivalence is obtained between the first condition in (39) and (41) with $\mathcal{I}_{F 2 C}$ replaced by $\mathcal{I}_{C 2 F}$. From Proposition 4 it follows that a penalty matrix $\Sigma_{h, t_{2}}$ can be found such that (40) is energy stable for any SBP preserving interpolation operators that satisfy (38).

In the special case when the coarse and fine grids are identical, the interpolation operators reduce to identity matrices. This corresponds to a multi-block formulation with colocated nodes, which often is beneficial from a computational point of view $[17,31,32]$. In this case, $P_{x,(u)}=P_{x,(v)}$ and the transmission condition (41) is trivially satisfied.

As an example of the temporal interpolation discussed above, consider a second order accurate case, where a coarse, uniform one-dimensional grid consisting of four grid points, and a similar but finer seven point grid is used. Let the step size be $h=2$ on the coarse grid and $h=1$ on the finer one. We may then choose

$$
P_{x,(u)}=2 \operatorname{diag}(1 / 2,1,1,1 / 2), \quad P_{x,(v)}=\operatorname{diag}(1 / 2,1,1,1,1,1,1 / 2)
$$

and the SBP preserving interpolation operator becomes [26]

$$
\mathcal{I}_{C 2 F}=\frac{1}{2}\left(\begin{array}{cccc}
2 & & & \\
1 & 1 & & \\
& 2 & & \\
& 1 & 1 & \\
& & 2 & \\
& & 1 & 1 \\
& & & 2
\end{array}\right) .
$$

Then, the eigenvalues of $P_{x,(u)}-I_{C 2 F}^{\top} P_{x,(v)} I_{C 2 F}$ to four decimal places are given by $\{0.5955,0.6047,1.1545,1.3953\}$, and hence $(41)$ is clearly satisfied. 


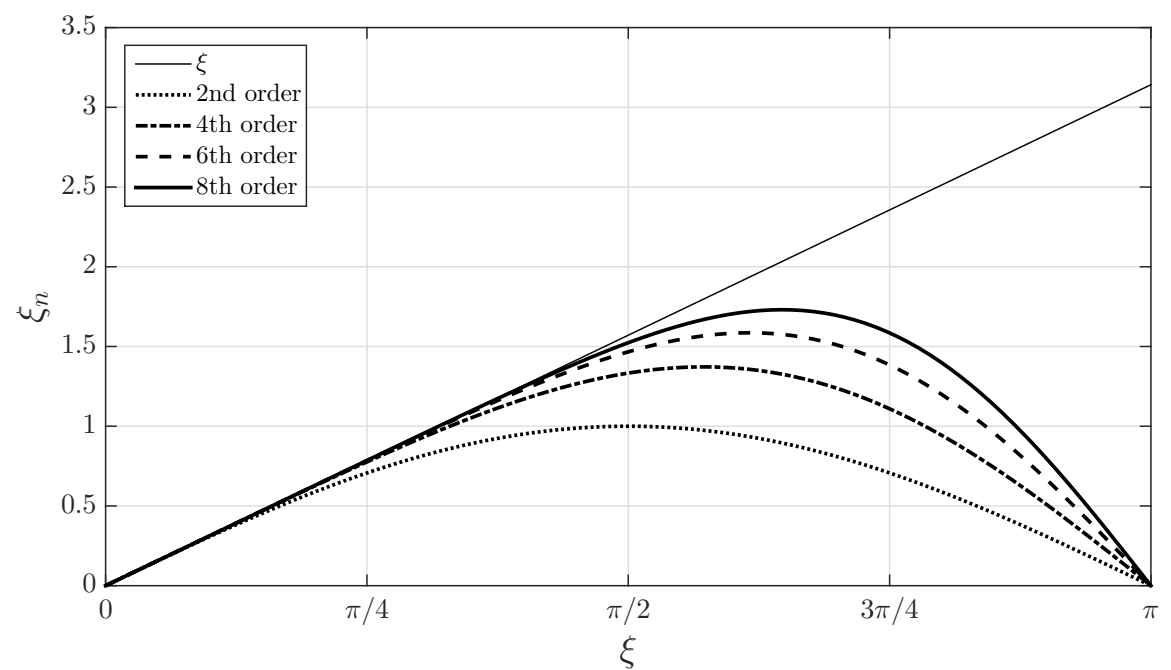

Figure 1: Numerical wavenumbers associated with central difference stencils.

\subsubsection{Explicit filters}

Errors are inevitably present in numerical simulations, even when the computations are well resolved, and are predominantly introduced at high wavenumbers. To see this, we may plot the numerical wavenumber $\xi_{n}$ associated with a given derivative approximation, against the analytic wavenumber $\xi$ associated with the actual derivative. This is done in Fig. 1 for central finite difference stencils of various orders. Evidently, the dispersion error $\xi-\xi_{n}$ grows as the wavenumber increases and, in fact, this growth is monotonic [33]. A plethora of finite difference stencils have been presented with reduced dispersion error for various ranges of wavenumbers; see [34] for a review and [35] for examples within the SBP-SAT framework. Yet, no difference stencil is accurate in the vicinity of $\xi=\pi$. Instead, filters designed to remove high wavenumbers from the computational domain may be applied.

Here, we will restrict our attention to the finite difference-type filters introduced in [36]. They take the form

$$
F=\left(\left(I_{x}+\alpha_{F} D_{x}^{(2 n)}\right),\right.
$$

where $D_{x}^{(2 n)}$ is an undivided (i.e. independent of the grid resolution) difference operator approximating the $2 n$th derivative. The scalar $\alpha_{F}=(-1)^{n} 2^{-2 n}$ ensures that the $\pi$-mode is removed. A candidate implementation of such a 
filter is

$$
\begin{aligned}
& \left(P_{t}^{-1} Q_{t} \otimes I_{x}\right) \mathbf{u}+I_{t} \otimes D(\mathbf{u})=-\left(P_{t}^{-1} E_{0} \otimes I_{x}\right)\left(I_{t} \otimes\left(\mathbf{u}_{0}-\mathbf{f}\right)\right) \\
& \left(P_{t}^{-1} Q_{t} \otimes I_{x}\right) \mathbf{v}+I_{t} \otimes D(\mathbf{v})=\left(P_{t}^{-1} E_{0} \otimes \Sigma_{h, t_{2}}\right)\left(I_{t} \otimes\left(\mathbf{v}_{0}-F \mathbf{u}_{n}\right)\right) .
\end{aligned}
$$

Here, the filter is applied after a given number of time steps determined by the dimension of the matrix $P_{t}$. Naturally this process is repeated at regular intervals.

With the implementation (42), the condition (16) becomes

$$
P_{x}-F^{\top} P_{x} F \geq 0 \text {. }
$$

It is easy to find examples of $P_{x}$ and $F$ where (43) is not satisfied. For simplicity, let the step size $h=1$. Then, the choices

$$
P_{x}=\left(\begin{array}{cccc}
\frac{1}{2} & & & \\
& 1 & & \\
& & 1 & \\
& & & \frac{1}{2}
\end{array}\right), \quad F=\frac{1}{4}\left(\begin{array}{cccc}
3 & 1 & & \\
1 & 2 & 1 & \\
& 1 & 2 & 1 \\
& & 1 & 3
\end{array}\right)
$$

result in $P_{x}-F^{\top} P_{x} F$ having eigenvalues $\{0.9375,0.5890,0.1250,-0.0265\}$, and hence an energy estimate cannot be obtained. We must therefore rescale the norm used to estimate the first equation in (42), and from (26) we obtain the scaled transmission condition

$$
\kappa P_{x}-F^{\top} P_{x} F \geq 0 .
$$

By Proposition 5 we may chose

$$
\kappa=\frac{\lambda_{\max }\left(F^{\top} P_{x} F\right)}{\lambda_{\min }\left(P_{x}\right)}=1.5531,
$$

which yields eigenvalues $\{1.4826,1.0813,0.4095,0.3108\}$ for $\kappa P_{x}-F^{\top} P_{x} F$, and an energy estimate is viable.

However, recall from (29) that if the filter is applied at $m$ regular intervals, the resulting energy estimate becomes

$$
\|\mathbf{v}\|_{P_{x}}^{2} \leq \kappa^{m}\|\mathbf{f}\|_{P_{x}}^{2}
$$

Already for $m=11$ we have $\kappa^{m}>100$, which is much too weak for most applications. The minimal value of $\kappa$ that yields an energy estimate is $\kappa=$ 1.0411 , for which $\kappa^{m}>100$ with $m=115$. For long-time simulations, this may still be too weak. 
Remark 8. This example illustrates that successful filtering may include a delicate balance between the need to remove high frequency oscillations (filter often) and the need to avoid possible growth (filter seldom).

Remark 9. Artificial dissipation operators are akin to filters applied at each time step and thus become an integral part of the spatial discretisation. See [37] for artificial dissipation operators in the SBP-SAT framework.

\section{Conclusion}

In this paper, we have introduced a general class of problems, referred to as transmission problems, describing the transmission of information between time-dependent problems governed by possibly different dynamics. No specific assumptions about the nature of the problems have been made, apart from them being maximally semi-bounded. A necessary and sufficient condition for energy boundedness has been obtained, which relates the transmission operator $\mathcal{X}$ to the norms in which the energy estimates are obtained before and after the time of transmission. This transmission condition is independent of whether a strong or weak formulation is used.

It has further been shown that the transmission condition is always satisfied if scaled norms are used. However, the choice of norms has a nonnegligible impact on the resulting energy estimate, and it is therefore desirable to chose optimal transmission operators.

Summation-by-Parts discretisations in time with a weak imposition of transmission conditions through SAT terms have been used to model discrete transmission problems. A necessary and sufficient condition for energy stability, analogous to the one obtained for the continuous problem, has been obtained. No assumptions about the spatial discretisations was made, apart from semi-boundedness. Thus, the presented results are general and applies to any problem with prescribed norms.

Transmission problems encompass many important problems as special cases; the list presented in this work is certainly not exhaustive. Here we have attempted to include examples with a wide spectrum of applications that, when possible, are independent of the underlying dynamics. Continuous transmission problems typically arise whenever one set of governing equations is replaced by another after some time. We have illustrated this by coupling the linearised Euler equations with the acoustic wave equation, and shown that with an appropriate choice of norms, an energy estimate is obtained. 
Further, a multi-grid implementation in connection to dual time-stepping has been considered. We cannot make claims about the stability of such implementations without making specific assumptions about the underlying problem and its stability norm. Nevertheless, we have obtained a necessary condition for an energy estimate, which depends only on the eigenvalues of the multi-grid update matrix, and is simple to verify.

A range of numerical techniques may also be modelled by the transmission problem. By using temporal interpolation between time-blocks, it is possible to adapt the time-step of a given SBP discretisation, which may have a significant impact on the performance of the scheme. We have shown that if the operators are SBP preserving, and further satisfy conditions related to the imposition of characteristic boundary conditions, then a SAT penalty matrix exists that yields an energy estimate. As a special case, colocated multi-block formulations in time trivially satisfy the condition necessary for a stable implementation.

Finally, we considered a numerical filter for which scaled norms must be used to obtain an energy estimate. It was shown that even the sharpest possible energy bound become very weak as the number of filtrations grow. This indicates that successful filtering may include a delicate balance between the need to remove high frequency oscillations (filter often) and the need to avoid possible growth (filter seldom).

\section{Appendix A. Proof of Proposition 5}

Proof. Let $R_{\kappa}=\kappa P_{1}-X^{\top} P_{2} X$ and let $\mathbf{y}$ be a real-valued vector. We must show that the quadratic form $\mathbf{y}^{\top} R_{\kappa} \mathbf{y} \geq 0$ for any choice of $\mathbf{y}$.

Recall that $P_{1}$ is symmetric positive definite. Hence, there is an orthogonal matrix $U$ such that $P_{1}=U^{\top} \Lambda U$, where $\Lambda$ is diagonal positive definite. Further, we may uniquely define the matrix $\Lambda^{1 / 2}$ as the square root of $\Lambda$. Consequently, we have

$$
\begin{aligned}
\mathbf{y}^{\top} R_{\kappa} \mathbf{y} & =\mathbf{y}^{\top}\left(\kappa P_{1}-X^{\top} P_{2} X\right) \mathbf{y} \\
& =\left(\Lambda^{1 / 2} U \mathbf{y}\right)^{\top}\left(\kappa I_{1}-\Lambda^{-1 / 2} U X^{\top} P_{2} X U^{\top} \Lambda^{-1 / 2}\right)\left(\Lambda^{1 / 2} U \mathbf{y}\right) \\
& =\mathbf{z}^{\top}(\kappa I_{1}-\underbrace{\Lambda^{-1 / 2} U X^{\top} P_{2} X U^{\top} \Lambda^{-1 / 2}}_{H}) \mathbf{z},
\end{aligned}
$$

where $\mathbf{z}=\left(\Lambda^{1 / 2} U \mathbf{y}\right)$. Note that $H$ is is symmetric positive semi-definite, and 
hence $\lambda_{\max }(H)$ coincides with the spectral norm of $H$;

$$
\lambda_{\max }(H)=\|H\|_{2}=\sup _{\|\mathbf{x}\|=1}\|H \mathbf{x}\|=\sqrt{\lambda_{\max }\left(H^{\top} H\right)},
$$

where $\|\cdot\|$ without subscripts denotes the Euclidean vector norm. Clearly it suffices to choose $\kappa \geq \lambda_{\max }(H)$ in order for the quadratic form (A.1) to be positive semi-definite. But

$$
\begin{aligned}
\lambda_{\max }(H) & =\|H\|_{2} \leq\left\|\Lambda^{-1 / 2}\right\|_{2}\left\|U^{\top}\right\|_{2}\left\|X^{\top} P_{2} X\right\|_{2}\|U\|_{2}\left\|\Lambda^{-1 / 2}\right\|_{2} \\
& =\frac{\left\|X^{\top} P_{2} X\right\|_{2}}{\lambda_{\min }\left(P_{1}\right)}=\frac{\lambda_{\max }\left(X^{\top} P_{2} X\right)}{\lambda_{\min }\left(P_{1}\right)},
\end{aligned}
$$

whence the proposition follows.

\section{Appendix B. Proof of Proposition 6}

Proof. Let $P_{1}=P_{2} \equiv P$ and assume that (26) holds. Since $P$ is positive definite it has a uniquely defined, positive definite square root, $P^{1 / 2}$. Multiplying (26) from the left and right by $P^{-1 / 2}$ gives by Sylvester's theorem

$$
0 \leq \kappa I-\left(P^{-1 / 2} X^{\top} P^{1 / 2}\right)\left(P^{1 / 2} X P^{-1 / 2}\right)=\kappa I-\hat{X}^{\top} \hat{X},
$$

where $\hat{X}=P^{1 / 2} X P^{-1 / 2}$. Clearly (B.1) implies that $\kappa \geq \lambda_{\max }\left(\hat{X}^{\top} \hat{X}\right) \geq$ $\lambda_{\max }^{2}(\hat{X})$ (see e.g. [38]). However, by similarity, the eigenvalues of $X$ are the same as those of $\hat{X}$, whence the proposition follows.

\section{References}

[1] B. Gustafsson, H.-O. Kreiss, J. Oliger, Time dependent problems and difference methods, volume 24, John Wiley \& Sons, 1995.

[2] H.-O. Kreiss, J. Lorenz, Initial-Boundary Value problems and the Navier-Stokes equations, SIAM, 2004.

[3] D. N. Arnold, F. Brezzi, B. Cockburn, L. D. Marini, Unified analysis of discontinuous galerkin methods for elliptic problems, SIAM journal on numerical analysis 39 (2002) 1749-1779.

[4] J. Nordström, A roadmap to well posed and stable problems in computational physics, Journal of Scientific Computing 71 (2017) 365-385. 
[5] H.-O. Kreiss, G. Scherer, Finite element and finite difference methods for hyperbolic partial differential equations, Aspects of Finite Elements in Partial Differential Equations, Academic Press, Inc. (1974).

[6] H.-O. Kreiss, G. Scherer, On the existence of energy estimates for difference approximations of hyperbolic systems, Technical report, Dept. of Scientific Computing, Uppsala University (1977).

[7] J. Nordström, M. Björck, Finite volume approximations and strict stability for hyperbolic problems, Applied numerical mathematics 38 (2001) 237-255.

[8] J. Nordström, K. Forsberg, C. Adamsson, P. Eliasson, Finite volume methods, unstructured meshes and strict stability for hyperbolic problems, Applied Numerical Mathematics 45 (2003) 453-473.

[9] M. Svärd, J. Nordström, Stability of finite volume approximations for the Laplacian operator on quadrilateral and triangular grids, Applied Numerical Mathematics 51 (2004) 101-125.

[10] M. Carpenter, D. Gottlieb, Spectral methods on arbitrary grids, Journal of Computational Physics 129 (1996) 74-86.

[11] G. J. Gassner, A skew-symmetric discontinuous Galerkin spectral element discretization and its relation to SBP-SAT finite difference methods, SIAM Journal on Scientific Computing 35 (2013) A1233-A1253.

[12] G. J. Gassner, A. R. Winters, D. A. Kopriva, Split form nodal discontinuous Galerkin schemes with Summation-by-Parts property for the compressible Euler equations, Journal of Computational Physics 327 (2016) 39-66.

[13] H. Ranocha, P. Öffner, T. Sonar, Summation-by-Parts operators for correction procedure via reconstruction, Journal of Computational Physics 311 (2016) 299-328.

[14] J. Nordström, T. Lundquist, Summation-by-Parts in time, Journal of Computational Physics 251 (2013) 487-499.

[15] T. Lundquist, J. Nordström, The SBP-SAT technique for initial value problems, Journal of Computational Physics 270 (2014) 86-104. 
[16] J. Nordström, T. Lundquist, Summation-by-Parts in time: the second derivative, SIAM Journal on Scientific Computing 38 (2016) A1561A1586.

[17] T. Lundquist, J. Nordström, Efficient fully discrete Summation-by-Parts schemes for unsteady flow problems, BIT Numerical Mathematics 56 (2016) 951-966.

[18] M. H. Carpenter, D. Gottlieb, S. Abarbanel, Time-stable boundary conditions for finite-difference schemes solving hyperbolic systems: methodology and application to high-order compact schemes, Journal of Computational Physics 111 (1994) 220-236.

[19] B. Cockburn, G. E. Karniadakis, C.-W. Shu, The development of discontinuous galerkin methods, in: Discontinuous Galerkin Methods, Springer, 2000, pp. 3-50.

[20] B. Gustafsson, High order difference methods for time dependent PDE, volume 38, Springer Science \& Business Media, 2007.

[21] D. C. D. R. Fernández, P. D. Boom, D. W. Zingg, A generalized framework for nodal first derivative summation-by-parts operators, Journal of Computational Physics 266 (2014) 214-239.

[22] M. Svärd, J. Nordström, Review of Summation-by-Parts schemes for initial-boundary-value problems, Journal of Computational Physics 268 (2014) 17-38.

[23] D. C. D. R. Fernández, J. E. Hicken, D. W. Zingg, Review of Summation-by-Parts operators with Simultaneous Approximation Terms for the numerical solution of partial differential equations, Computers \& Fluids 95 (2014) 171-196.

[24] S. Abarbanel, D. Gottlieb, Optimal time splitting for two-and threedimensional navier-stokes equations with mixed derivatives, Journal of Computational Physics 41 (1981) 1-33.

[25] W. L. Briggs, V. E. Henson, S. F. McCormick, A multigrid tutorial, SIAM, 2000. 
[26] A. A. Ruggiu, P. Weinerfelt, J. Nordström, A new multigrid formulation for high order finite difference methods on Summation-by-Parts form, Technical Report LiTH-MAT-R, ISSN 0348-2960; 2017:08, Linköping University, 2017.

[27] G. Chesshire, W. D. Henshaw, Composite overlapping meshes for the solution of partial differential equations, Journal of Computational Physics 90 (1990) 1-64.

[28] N. A. Petersson, B. Sjögreen, Stable grid refinement and singular source discretization for seismic wave simulations, Communications in Computational Physics, vol. 8, no. 5, May 31, 2010, pp. 1074-1110 8 (2009).

[29] K. Mattsson, M. H. Carpenter, Stable and accurate interpolation operators for high-order multiblock finite difference methods, SIAM Journal on Scientific Computing 32 (2010) 2298-2320.

[30] J. E. Kozdon, L. C. Wilcox, Stable coupling of nonconforming, highorder finite difference methods, SIAM Journal on Scientific Computing 38 (2016) A923-A952.

[31] P. Diener, E. N. Dorband, E. Schnetter, M. Tiglio, Optimized high-order derivative and dissipation operators satisfying summation by parts, and applications in three-dimensional multi-block evolutions, Journal of Scientific Computing 32 (2007) 109-145.

[32] J. Nordström, J. Gong, E. Van der Weide, M. Svärd, A stable and conservative high order multi-block method for the compressible navierstokes equations, Journal of Computational Physics 228 (2009) 90209035 .

[33] V. Linders, J. Nordström, Uniformly best wavenumber approximations by spatial central difference operators, Journal of Computational Physics 300 (2015) 695-709.

[34] D. W. Zingg, Comparison of high-accuracy finite-difference methods for linear wave propagation, SIAM J. Sci. Comput. 22 (2000) 476-502.

[35] V. Linders, M. Kupiainen, J. Nordström, Summation-by-Parts operators with minimal dispersion error for coarse grid flow calculations, Journal of Computational Physics 340 (2017) 160-176. 
[36] C. A. Kennedy, M. H. Carpenter, Comparison of several numerical methods for simulation of compressible shear layers, in: NASA technical paper 3438, Langley research.

[37] K. Mattsson, M. Svärd, J. Nordström, Stable and accurate artificial dissipation, Journal of Scientific Computing 21 (2004) 57-79.

[38] P. Lancaster, Theory of matrices, Academic Press, Inc., 1969. 